\title{
External Ear
}

National Cancer Institute

\section{Source}

National Cancer Institute. External Ear. NCI Thesaurus. Code C12292.

The external part of the ear. 